\title{
Ring Signatures: Stronger Definitions, and Constructions without Random Oracles
}

\author{
Adam Bender ${ }^{1}$, Jonathan Katz ${ }^{1, \star}$, and Ruggero Morselli ${ }^{1, \star \star}$ \\ Department of Computer Science, University of Maryland \\ \{bender, jkatz, ruggero\}@cs. umd.edu
}

\begin{abstract}
Ring signatures, first introduced by Rivest, Shamir, and Tauman, enable a user to sign a message so that a ring of possible signers (of which the user is a member) is identified, without revealing exactly which member of that ring actually generated the signature. In contrast to group signatures, ring signatures are completely "ad-hoc" and do not require any central authority or coordination among the various users (indeed, users do not even need to be aware of each other); furthermore, ring signature schemes grant users fine-grained control over the level of anonymity associated with any particular signature.

This paper has two main areas of focus. First, we examine previous definitions of security for ring signature schemes and suggest that most of these prior definitions are too weak, in the sense that they do not take into account certain realistic attacks. We propose new definitions of anonymity and unforgeability which address these threats, and then give separation results proving that our new notions are strictly stronger than previous ones. Next, we show two constructions of ring signature schemes in the standard model: one based on generic assumptions which satisfies our strongest definitions of security, and a second, more efficient scheme achieving weaker security guarantees and more limited functionality. These are the first constructions of ring signature schemes that do not rely on random oracles or ideal ciphers.
\end{abstract}

\section{Introduction}

Ring signatures enable a user to sign a message so that a "ring" of possible signers (of which the user is a member) is identified, without revealing exactly which member of that ring actually generated the signature. This notion was first formally introduced by Rivest, Shamir, and Tauman [20], and ring signatures - along with the related notion of ring/ad-hoc identification schemes - have been studied extensively since then $[5,19,1,23,16,11,22,18,2]$. Ring signatures are related, but incomparable, to the notion of group signatures [6]. On the one hand, group signatures have the additional feature that the anonymity of a signer can be revoked (i.e., the signer can be traced) by a designated group manager.

\footnotetext{
* This research was supported in part by NSF Trusted Computing Grants \#0310499 and \#0310751, NSF-ITR \#0426683, and NSF CAREER award \#0447075.

** Supported by NSF Trusted Computing Grant \#0310499 and NSF-ITR \#0426683.
} 
On the other hand, ring signatures allow greater flexibility: no centralized group manager or coordination among the various users is required (indeed, users may be unaware of each other at the time they generate their public keys), rings may be formed completely "on-the-fly" and in an ad-hoc manner, and users are given fine-grained control over the level of anonymity associated with any particular signature (via selection of an appropriate ring).

Ring signatures naturally lend themselves to a variety of applications which have been suggested already in previous work (see especially $[20,19,11,2]$ ). The original motivation was to allow secrets to be leaked anonymously. Here, for example, a high-ranking government official can sign information with respect to the ring of all similarly high-ranking officials; the information can then be verified as coming from someone reputable without exposing the actual signer. Ring signatures can also be used to provide a member of a certain class of users access to a particular resource without explicitly identifying this member; note that there may be cases when third-party verifiability is required (e.g., to prove that the resource has been accessed) and so ring signatures, rather than adhoc identification schemes, are needed. Finally, we mention the application to designated-verifier signatures [17] especially in the context of e-mail. Here, ring signatures enable the sender of an e-mail to sign the message with respect to the ring containing the sender and the receiver; the receiver is then assured that the e-mail originated from the sender but cannot prove this to any third party. We remark that for this latter application it is sufficient to use a ring signature scheme which supports only rings of size two. See also [7] for another proposed application of ring signatures which support only rings of size two.

\subsection{Our Contributions in Relation to Previous Work}

This paper focuses on both definitions and constructions. We summarize our results in each of these areas, and relate them to prior work.

Definitions of security. Prior work on ring signature/identification schemes provides definitions of security that are either rather informal or seem (to us) unnaturally weak, in that they do not address what seem to be valid security concerns. One example is the failure to consider the possibility of adversariallychosen public keys. Specifically, both the anonymity and unforgeability definitions in most prior work assume that honest users always sign with respect to rings consisting entirely of honestly-generated public keys; no security is provided if users sign with respect to a ring containing even one adversarially-generated public key. Clearly, however, a scheme which is not secure in the latter case is of limited use; this is especially true since rings are constructed in an ad-hoc fashion using keys of (possibly unknown) users which are not validated as being correctly constructed by any central authority. We formalize security against such attacks (as well as others), and show separation results proving that our definitions are strictly stronger than those considered in previous work. In addition to the new, strong definitions we present, the hierarchy of definitions we give is useful for characterizing the security of ring signature constructions. 
Constructions. We show two constructions of ring signature schemes which are proven secure in the standard model. We stress that these are the first such constructions, as all previous constructions of which we are aware rely on random oracles/ideal ciphers. ${ }^{1}$ It is worth remarking that ring identification schemes are somewhat easier to construct (using, e.g., techniques from [9]); ring signatures can then easily be derived from such schemes using the Fiat-Shamir methodology in the random oracle model [14]. This approach, however, is no longer viable (at least, based on our current understanding) when working in the standard model.

Our first construction is based on generic assumptions, and satisfies the strongest definitions of anonymity and unforgeability considered here. This construction is inspired by the generic construction of group signatures due to Bellare, et al. [3] and, indeed, the constructions share some similarities at a high level. However, a number of subtleties arise in our context that do not arise in the context of group signatures, and the construction given in [3] does not immediately lend itself to a ring signature scheme. Two issues in particular that we need to deal with are the fact that we have no central group manager to issue "certificates" as in [3], and that we additionally need to take into account the possibility of adversarially-generated public keys as discussed earlier (this is not a concern in [3] where there is only a single group public key published by a (semi-)trusted group manager).

Our second construction is more efficient than the first, but relies on specific number-theoretic assumptions. Furthermore, it provides more limited functionality and security guarantees than our first construction; most limiting is that it only supports rings of size two. Such a scheme is still useful for certain applications (as discussed earlier); furthermore, constructing an efficient 2-user ring signature scheme without random oracles seems difficult, as we still do not have the Fiat-Shamir methodology available in our toolbox. This second scheme is based on the (standard) signature scheme recently proposed by Waters [21] in the context of ID-based encryption; in fact, we reduce the security of our scheme directly to the security of his scheme.

\section{Preliminaries}

We use the standard definitions of public-key encryption schemes and semantic security, signature schemes and existential unforgeability under adaptive chosenmessage attacks, and computational indistinguishability. In this paper we will assume public-key encryption schemes for which, with all but negligible probability over $(p k, s k)$ generated at random using the specified key generation algorithm, $\operatorname{Dec}_{s k}\left(\operatorname{Enc}_{p k}(M)\right)=M$ holds with probability 1 .

\footnotetext{
${ }^{1}$ Although $\mathrm{X} u$, Zhang, and Feng [22] claim a ring signature scheme in the standard model based on specific assumptions, their proof was later found to be flawed (personal communication from J. Xu, March 2005). Concurrently to our work, Chow, Liu and Yuen [8] show a ring signature scheme that they prove secure in the standard model (for rings of constant size) based on a new number-theoretic assumption.
} 
We will also use the notion of a $Z A P$, which is a 2-round, public-coin, witnessindistinguishable proof system for any language in $\mathcal{N} \mathcal{P}$ (the formal definition is given in Appendix A). ZAPs were introduced by Dwork and Naor [12], who show that ZAPs can be constructed based on trapdoor permutations. For notational purposes, we represent a ZAP by a triple $(\ell, \mathcal{P}, \mathcal{V})$ such that $(1)$ the initial message $r$ from the verifier has length $\ell(k)$ (where $k$ is the security parameter); (2) the prover $\mathcal{P}$, on input the verifier-message $r$, statement $x$, and witness $w$, outputs $\pi \leftarrow \mathcal{P}_{r}(x, w)$; finally, (3) $\mathcal{V}_{r}(x, \pi)$ outputs 1 or 0 , indicating acceptance or rejection of the proof.

\section{Definitions}

We begin by presenting the functional definition of a ring signature scheme. We refer to an ordered list $R=\left(P K_{1}, \ldots, P K_{n}\right)$ of public keys as a ring, and let $R[i]=P K_{i}$. We will also freely use set notation, and say, e.g., that $P K \in R$ if there exists an index $i$ such that $R[i]=P K$. We will always assume, without loss of generality, that the keys in a ring are ordered lexicographically.

Definition 1 (Ring signature). A ring signature scheme is a triple of PPT algorithms (Gen, Sign, Vrfy) that, respectively, generate keys for a user, sign a message, and verify the signature of a message. Formally:

- Gen $\left(1^{k}\right)$, where $k$ is a security parameter, outputs a public key PK and secret key $S K$.

- $\operatorname{Sign}_{s, S K}(M, R)$ outputs a signature $\sigma$ on the message $M$ with respect to the ring $R=\left(P K_{1}, \ldots, P K_{n}\right)$. We assume the following: (1) $(R[s], S K)$ is a valid key-pair output by Gen; (2) $|R| \geq 2$ (since a ring signature scheme is not intended ${ }^{2}$ to serve as a standard signature scheme); and (3) each ${ }^{3}$ public key in the ring is distinct.

- $\operatorname{Vrfy}_{R}(M, \sigma)$ verifies a purported signature $\sigma$ on a message $M$ with respect to the ring of public keys $R$.

We require the following completeness condition to hold: for any integer $k$, any $\left\{\left(P K_{i}, S K_{i}\right)\right\}_{i=1}^{n}$ output by $\operatorname{Gen}\left(1^{k}\right)$, any $s \in[n]$, and any $M$, we have $\operatorname{Vrfy}_{R}\left(M, \operatorname{Sign}_{s, S K_{s}}(M, R)\right)=1$ where $R=\left(P K_{1}, \ldots, P K_{n}\right)$.

$A$ c-user ring signature scheme is a variant of the above that only supports rings of fixed size c (i.e., the Sign and Vrfy algorithms only take as input rings $R$ for which $|R|=c$, and correctness is only required to hold for such rings).

To improve readability, we will generally omit the input " $s$ " to the signing algorithm (and simply write $\sigma \leftarrow \operatorname{Sign}_{S K}(M, R)$ ), with the understanding that the signer can determine an index $s$ for which $S K$ is the secret key corresponding

\footnotetext{
${ }^{2}$ Furthermore, it is easy to modify any ring signature scheme to allow signatures with $|R|=1$ by including a special key for just that purpose.

3 This is without loss of generality, since the signer/verifier can simply take the subring of distinct keys in $R$ and correctness is unchanged.
} 
to public key $R[s]$. Strictly speaking, there may not be a unique such $s$ when $R$ contains incorrectly-generated keys; in real-world usage of a ring signature scheme, though, a signer will certainly be able to identify their public key.

A ring signature scheme is used as follows: At various times, some collection of users runs the key generation algorithm Gen to generate public and secret keys. We stress that no coordination among these users is assumed or required. When a user with secret key $S K$ wishes to generate an anonymous signature on a message $M$, he chooses a ring $R$ of public keys which includes his own, computes $\sigma \leftarrow \operatorname{Sign}_{S K}(M, R)$ and outputs $(\sigma, R)$. (In such a case, we will refer to the holder of $S K$ as the signer of the message and to the holders of the other public keys in $R$ as the non-signers.) Anyone can now verify that this signature was generated by someone holding a key in $R$ by running $\operatorname{Vrfy}_{R}(M, \sigma)$.

We remark that although our functional definition of a ring signature scheme (cf. Def. 1) requires users to generate keys specifically for that purpose (in contrast to the requirements of $[1,2]$ ), our first construction can be easily modified to work with any ring of users as long as they each have a public key for both encryption and signing (see Sect. 5).

As discussed in the Introduction, ring signatures must satisfy two independent notions of security: anonymity and unforgeability. There are various ways each of these notions can be defined (and various ways these notions have been defined in the literature); we present our definitions in Sections 3.1 and 3.2, and compare them in Sect. 4.

\subsection{Definitions of Anonymity}

The anonymity condition requires, informally, that an adversary not be able to tell which member of a ring generated a particular signature. ${ }^{4}$ We begin with a basic definition of anonymity which is already stronger than that considered in most previous work in that we give the adversary access to a signing oracle (this results in a stronger definition even in the case of unconditional anonymity).

Definition 2 (Basic anonymity). Given a ring signature scheme (Gen, Sign, Vrfy), a polynomial $n(\cdot)$, and a PPT adversary $\mathcal{A}$, consider the following game:

1. Key pairs $\left\{\left(P K_{i}, S K_{i}\right)\right\}_{i=1}^{n(k)}$ are generated using $\operatorname{Gen}\left(1^{k}\right)$, and the set of public keys $S \stackrel{\text { def }}{=}\left\{P K_{i}\right\}_{i=1}^{n(k)}$ is given to $\mathcal{A}$.

2. $\mathcal{A}$ is given access (throughout the entire game) to an oracle Osign $(\cdot, \cdot, \cdot)$ such that $\operatorname{Osign}(s, M, R)$ returns $\operatorname{Sign}_{S K_{s}}(M, R)$, where we require $R \subseteq S$ and $P K_{s} \in R$.

3. $\mathcal{A}$ outputs a message $M$, distinct indices $i_{0}, i_{1}$, and a ring $R \subseteq S$ for which $P K_{i_{0}}, P K_{i_{1}} \in R$. A random bit $b$ is chosen, and $\mathcal{A}$ is given the signature $\sigma \leftarrow \operatorname{Sign}_{S K_{i_{b}}}(M, R)$.

\footnotetext{
${ }^{4}$ All the anonymity definitions that follow can be phrased in either a computational or an unconditional sense (where, informally, in the former case anonymity holds for polynomial-time adversaries while in the latter case anonymity holds even for allpowerful adversaries). For simplicity, we only present the computational versions.
} 


\section{The adversary outputs a bit $b^{\prime}$, and succeeds if $b^{\prime}=b$.}

(Gen, Sign, Vrfy) achieves basic anonymity if, for any PPT $\mathcal{A}$ and any polynomial $n(\cdot)$, the success probability of $\mathcal{A}$ in the above game is negligibly close to $1 / 2$.

(Some previous papers consider a variant of the above in which the adversary is given a signature computed by a randomly-chosen member of $R$, and should be unable to guess the actual signer with probability better than $1 /|R|+\operatorname{negl}(k)$. A hybrid argument shows that such a variant is equivalent to the above.)

Unfortunately, the above definition of basic anonymity leaves open the possibility of the following attack: (1) an adversary generates public keys in some arbitrary manner (which may possibly depend on the public keys of the honest users), and then (2) a legitimate signer generates a signature with respect to a ring containing some of these adversarially-generated public keys. The definition above offers no protection in this case! This attack, considered also in [19] (in a slightly different context) is quite realistic since, by their very nature, ring signatures are intended to be used in settings where there is not necessarily any central authority checking validity of public keys. This motivates the following, stronger definition:

Definition 3 (Anonymity w.r.t. adversarially-chosen keys). Given a ring signature scheme (Gen, Sign, Vrfy), a polynomial $n(\cdot)$, and a PPT adversary $\mathcal{A}$, consider the following game:

1. As in Definition 2.

2. As in Definition 2, except that we no longer require $R \subseteq S$.

3. As in Definition 2, except that we no longer require $R \subseteq S$.

4. The adversary outputs a bit $b^{\prime}$, and succeeds if $b^{\prime}=b$.

(Gen, Sign, Vrfy) achieves anonymity w.r.t. adversarially-chosen keys if for any $\mathrm{PPT} \mathcal{A}$ and polynomial $n(\cdot)$, the success probability of $\mathcal{A}$ in the above game is negligibly close to $1 / 2$.

The above definition only guarantees anonymity of a particular signature as long as there are at least two honest users in the ring. In some sense this is inherent, since if an honest signer $U$ chooses a ring in which all other public keys (i.e., except for the public key of $U$ ) are owned by an adversary, then that adversary "knows" that $U$ must be the signer (since the adversary did not generate the signature itself).

A weaker requirement one might consider when the signer $U$ is the only honest user in the ring is that the adversary should be unable to prove to a third party that $U$ generated the signature (we call this an attribution attack). Preventing such an attack in general seems to require the involvement of a trusted party (or at least a common random string), something we would like to avoid. We instead define a slightly weaker notion which, informally, can be viewed as offering some protection against attribution attacks as long as at least one other user $U^{\prime}$ in the ring generated her public key honestly. The honest users 
in the ring (other ${ }^{5}$ than $U$ ), however, may later cooperate with the adversary by revealing their secret keys. (Actually, we even allow these users to reveal the randomness ${ }^{6}$ used to generate their secret keys.) Note that this also ensures some measure of security in case secret keys are exposed or stolen.

In addition to the above, we consider also the stronger variant in which the secret keys of all honest users (i.e., including $U$ ) are exposed. This parallels (in fact, is stronger than) the anonymity definition given by Bellare, et al. in the context of group signatures [3]. For simplicity, we also protect against adversariallychosen keys, although one could consider the weaker definition which does not.

Definition 4 (Anonymity against attribution attacks/full key exposure). Given (Gen, Sign, $\operatorname{Vrfy}), n(\cdot)$, and $\mathcal{A}$ as in Definition 3, consider the following game:

1. For $i=1$ to $n(k)$, generate $\left(P K_{i}, S K_{i}\right) \leftarrow \mathrm{Gen}\left(1^{k} ; \omega_{i}\right)$ for randomly-chosen $\omega_{i}$. Give to $\mathcal{A}$ the set of public keys $\left\{P K_{i}\right\}_{i=1}^{n(k)}$. The adversary $\mathcal{A}$ is also given access to a signing oracle as in Definition 3.

2. $\mathcal{A}$ outputs a message $M$, distinct indices $i_{0}, i_{1}$, and a ring $R$ for which $P K_{i_{0}}, P K_{i_{1}} \in R$. Adversary $\mathcal{A}$ is given $\left\{\omega_{i}\right\}_{i \neq i_{0}}$. Furthermore, a random bit $b$ is chosen and $\mathcal{A}$ is given $\sigma \leftarrow \operatorname{Sign}_{S K_{i_{b}}}(M, R)$.

3. The adversary outputs a bit $b^{\prime}$, and succeeds if $b^{\prime}=b$.

(Gen, Sign, Vrfy) achieves anonymity against attribution attacks if, for any PPT $\mathcal{A}$ and polynomial $n(\cdot)$, the success probability of $\mathcal{A}$ in the above game is negligibly close to $1 / 2$. If, in the second step, $\mathcal{A}$ is instead given $\left\{\omega_{i}\right\}_{i=1}^{n(k)}$ then we say (Gen, Sign, Vrfy) achieves anonymity against full key exposure.

Linkability. Another desideratum of a ring signature scheme is that it be unlinkable; that is, it be infeasible to determine whether two signatures (possibly generated with respect to different rings) were generated by the same signer. As in [3], all our definitions imply (appropriate variants of) unlinkability.

\subsection{Definitions of Unforgeability}

The intuitive notion of unforgeability is, as usual, that an adversary should be unable to output $(R, M, \sigma)$ such that $\operatorname{Vrfy}_{R}(M, \sigma)=1$ unless either (1) the adversary explicitly knows a secret key corresponding to one of the public keys in $R$, or (2) a user whose public key is in $R$ explicitly signed $M$ previously (with respect to the same ring $R$ ). Some subtleties arise, however, when defining what it means to allow the adversary a chosen-message attack on the scheme. Many previous works (e.g., [20]), assume a definition like the following:

\footnotetext{
5 The idea is that everyone in the ring is trying to "frame" $U$, but $U$ is (naturally) refusing to divulge her secret key. Although this itself might arouse suspicion, the point is that it still cannot be proved - in court, say - that $U$ was the signer.

${ }^{6}$ This ensures security when erasure cannot be guaranteed, or when it cannot be guaranteed that all users will comply with the directive to erase their random coins.
} 
Definition 5 (Unforgeability against fixed-ring attacks). A ring signature scheme (Gen, Sign, Vrfy) is unforgeable against fixed-ring attacks if for any PPT adversary $\mathcal{A}$ and for any polynomial $n(\cdot)$, the probability that $\mathcal{A}$ succeeds in the following game is negligible:

1. Key pairs $\left\{\left(P K_{i}, S K_{i}\right)\right\}_{i=1}^{n(k)}$ are generated using $\operatorname{Gen}\left(1^{k}\right)$, and the set of public keys $R \stackrel{\text { def }}{=}\left\{P K_{i}\right\}_{i=1}^{n(k)}$ is given to $\mathcal{A}$.

2. $\mathcal{A}$ is given access to a signing oracle OSign $(\cdot, \cdot)$, where $\operatorname{OSign}(s, M)$ outputs $\operatorname{Sign}_{S K_{s}}(M, R)$.

3. $\mathcal{A}$ outputs $\left(M^{*}, \sigma^{*}\right)$, and succeeds if $\operatorname{Vrfy}_{R}\left(M^{*}, \sigma^{*}\right)=1$ and also $\mathcal{A}$ never made a query of the form $\operatorname{Osign}\left(\star, M^{*}\right)$.

Note that not only is $\mathcal{A}$ restricted to making signing queries with respect to the full ring $R$, but its forgery is required to verify with respect to $R$ as well. The following stronger, and more natural, definition was used in, e.g., [1]:

Definition 6 (Unforgeability against chosen-subring attacks). A ring signature scheme (Gen, Sign, Vrfy) is unforgeable against chosen-subring attacks if for any PPT adversary $\mathcal{A}$ and for any polynomial $n(\cdot)$, the probability that $\mathcal{A}$ succeeds in the following game is negligible:

1. Key pairs $\left\{\left(P K_{i}, S K_{i}\right)\right\}_{i=1}^{n(k)}$ are generated using $\mathrm{Gen}\left(1^{k}\right)$, and the set of public keys $S \stackrel{\text { def }}{=}\left\{P K_{i}\right\}_{i=1}^{n(k)}$ is given to $\mathcal{A}$.

2. $\mathcal{A}$ is given access to a signing oracle $\operatorname{OSign}(\cdot, \cdot, \cdot)$, where $\operatorname{OSign}(s, M, R)$ outputs $\operatorname{Sign}_{S K_{s}}(M, R)$ and we require that $R \subseteq S$ and $P K_{s} \in R$.

3. $\mathcal{A}$ outputs $\left(R^{*}, M^{*}, \sigma^{*}\right)$, and succeeds if $R^{*} \subseteq S, \operatorname{Vrfy}_{R^{*}}\left(M^{*}, \sigma^{*}\right)=1$, and $\mathcal{A}$ never queried $\left(\star, M^{*}, R^{*}\right)$ to its signing oracle.

While the above definition is an improvement, it still leaves open the possibility of an attack whereby honest users are "tricked" into generating signatures using rings containing adversarially-generated public keys. (Such an attack was also previously suggested by $[19,18]$.) The following definition takes this into account as well as (for completeness) an adversary who adaptively corrupts honest participants and obtains their secret keys. Since either of these attacks may be viewed as the outcome of corrupting an "insider," we use this terminology. ${ }^{7}$

Definition 7 (Unforgeability w.r.t. insider corruption). A ring signature scheme (Gen, Sign, Vrfy) is unforgeable w.r.t. insider corruption if for any PPT adversary $\mathcal{A}$ and for any polynomial $n(\cdot)$, the probability that $\mathcal{A}$ succeeds in the following game is negligible:

1. Key pairs $\left\{\left(P K_{i}, S K_{i}\right)\right\}_{i=1}^{n(k)}$ are generated using $\operatorname{Gen}\left(1^{k}\right)$, and the set of public keys $S \stackrel{\text { def }}{=}\left\{P K_{i}\right\}_{i=1}^{n(k)}$ is given to $\mathcal{A}$.

\footnotetext{
${ }^{7}$ We are aware that, technically speaking, there are not really any "insiders" in the context of ring signatures.
} 
2. $\mathcal{A}$ is given access to a signing oracle $\operatorname{OSign}(\cdot, \cdot, \cdot)$, where $\operatorname{OSign}(s, M, R)$ outputs $\operatorname{Sign}_{s, S K_{s}}(M, R)$ and we require that $P K_{s} \in R$.

3. $\mathcal{A}$ is also given access to a corrupt oracle $\operatorname{Corrupt}(\cdot)$, where $\operatorname{Corrupt}(i)$ outputs $S K_{i}$.

4. $\mathcal{A}$ outputs $\left(R^{*}, M^{*}, \sigma^{*}\right)$, and succeeds if $\operatorname{Vrfy}_{R^{*}}\left(M^{*}, \sigma^{*}\right)=1, \mathcal{A}$ never queried $\left(\star, M^{*}, R^{*}\right)$, and $R^{*} \subseteq S \backslash C$, where $C$ is the set of corrupted users.

We remark that Herranz [15] considers, albeit informally, a definition intermediate between our Definitions 6 and 7 in which corruptions of honest players are allowed but adversarially-chosen public keys are not explicitly mentioned.

\section{Separations Between the Security Definitions}

In the previous section, we presented various definitions of anonymity and unforgeability. Here, we show that these definitions are in fact distinct, in the sense that there exist (under certain assumptions) schemes satisfying a weaker definition but not a stronger one. First, we show separations for the definitions of anonymity, considering in each case a scheme simultaneously satisfying the strongest definition of unforgeability (all proofs appear in the full version [4]):

Claim 1 If there exists a scheme which achieves basic anonymity and is unforgeable w.r.t. insider corruption, then there exists a scheme which achieves these same properties but which is not anonymous w.r.t. adversarially-chosen keys.

Claim 2 If there exists a scheme which is anonymous w.r.t. adversariallychosen keys and is unforgeable w.r.t. insider corruption, then there exists a scheme which achieves these same properties but which is not anonymous against attribution attacks.

We also show separations for the definitions of unforgeability, considering now schemes which simultaneously achieve the strongest definition of anonymity:

Claim 3 If there exists a scheme which is anonymous against full key exposure and unforgeable w.r.t. insider corruption, then there exists a scheme which is anonymous against full key exposure and unforgeable against fixed-ring attacks, but not unforgeable against chosen-subring attacks.

In contrast to the rest of the claims, the assumption in the above claim is not minimal. We remark that the scheme of [16] serves as a natural example of a scheme that is unforgeable against fixed-ring attacks, but which is not unforgeable against chosen-subring attacks (in the random oracle model); this was subsequently fixed in [15]. We defer a detailed discussion to the full version [4].

Claim 4 If there exists a scheme which is anonymous against full key exposure and unforgeable against chosen-subring attacks, then there exists a scheme achieving these same properties which is not unforgeable w.r.t. insider corruption. 


\section{Ring Signatures Based on General Assumptions}

We now describe our construction of a ring signature scheme that satisfies the strongest of our proposed definitions, and is based on general assumptions. In what follows, we let (EGen, Enc, Dec) be a semantically-secure public-key encryption scheme, let $\left(\mathrm{Gen}^{\prime}, \mathrm{Sign}^{\prime}, \mathrm{Vrfy}^{\prime}\right)$ be a (standard) signature scheme, and let $(\ell, \mathcal{P}, \mathcal{V}$ ) be a ZAP (for an $\mathcal{N} \mathcal{P}$-language that will become clear once we describe the scheme). We denote by $C^{*} \leftarrow \operatorname{Enc}_{R_{E}}^{*}(m)$ the probabilistic algorithm that takes as input a set of encryption public keys $R_{E}=\left\{p k_{E, 1}, \ldots, p k_{E, n}\right\}$ and a message $m$, and does the following: it first chooses random $s_{1}, \ldots, s_{n-1} \in\{0,1\}^{|m|}$ and then outputs:

$C^{*}=\left(\operatorname{Enc}_{p k_{E, 1}}\left(s_{1}\right), \operatorname{Enc}_{p k_{E, 2}}\left(s_{2}\right), \cdots, \operatorname{Enc}_{p k_{E, n-1}}\left(s_{n-1}\right), \operatorname{Enc}_{p k_{E, n}}\left(m \oplus \bigoplus_{j=1}^{n-1} s_{j}\right)\right)$.

Note that, informally, encryption using Enc* is semantically secure as long as at least one of the corresponding secret keys is unknown.

The idea of our construction is the following. Each user has an encryption key pair $\left(p k_{E}, s k_{E}\right)$ and a standard signature key pair $\left(p k_{S}, s k_{S}\right)$. To generate a ring signature with respect to a ring $R$ of $n$ users, the signer produces a standard signature $\sigma^{\prime}$ with her signing key. Next, the signer produces ciphertexts $C_{1}^{*}, \ldots, C_{n}^{*}$ using the Enc ${ }^{*}$ algorithm and the set $R_{E}$ of all the encryption public keys in the ring; one of these ciphertexts will be an encryption of $\sigma^{\prime}$. Finally, the signer produces a proof $\pi$, using the ZAP, that one of the ciphertexts is an encryption of a valid signature on the message with respect to the signature public key of one of the ring members.

Toward a formal description, let $L$ denote the $\mathcal{N P}$ language:

$$
\left\{\left(p k_{S}, M, R_{E}, C^{*}\right): \exists \sigma, \omega \text { s.t. } C^{*}=\operatorname{Enc}_{R_{E}}^{*}(\sigma ; \omega) \bigwedge \operatorname{Vrfy}_{p k_{S}}^{\prime}(M, \sigma)=1\right\} ;
$$

i.e., $\left(p k_{S}, M, R_{E}, C^{*}\right) \in L$ if $C^{*}$ is an encryption (using Enc $R_{E}^{*}$ ) of a valid signature of $M$ with respect to the public key $p k_{S}$. We now give the details of our construction, which is specified by the key-generation algorithm Gen, the ring signing algorithm Sign, and the ring verification algorithm Vrfy:

$\underline{\operatorname{Gen}\left(1^{k}\right)}$ :

1. Generate signing key pair $\left(p k_{S}, s k_{S}\right) \leftarrow \operatorname{Gen}^{\prime}\left(1^{k}\right)$.

2. Generate encryption key pair $\left(p k_{E}, s k_{E}\right) \leftarrow \operatorname{Gen}\left(1^{k}\right)$ and erase $s k_{E}$.

3. Choose an initial ZAP message $r \leftarrow\{0,1\}^{\ell(k)}$.

4. Output the public key $P K=\left(p k_{S}, p k_{E}, r\right)$, and the secret key $S K=s k_{S}$.

$\underline{\operatorname{Sign}_{i^{*}, S K_{i}}\left(M,\left(P K_{1}, \ldots, P K_{n}\right)\right)}:$

1. Parse each $P K_{i}$ as $\left(p k_{S, i}, p k_{E, i}, r_{i}\right)$, and parse $S K_{i^{*}}$ as $s k_{S, i^{*}}$. Set $R_{E}:=$ $\left\{p k_{E, 1}, \ldots, p k_{E, n}\right\}$. 
2. Set $M^{*}:=M\left|P K_{1}\right| \cdots \mid P K_{n}$, where "|" denotes concatenation. Compute the signature $\sigma_{i^{*}}^{\prime} \leftarrow \operatorname{Sign}_{s k_{S, i^{*}}}^{\prime}\left(M^{*}\right)$.

3. Choose random coins $\omega_{1}, \ldots, \omega_{n}$ and: (1) compute $C_{i^{*}}^{*}=\operatorname{Enc}_{R_{E}}^{*}\left(\sigma_{i^{*}}^{\prime} ; \omega_{i^{*}}\right)$ and (2) for $i \in\{1, \ldots, n\} \backslash\left\{i^{*}\right\}$, compute ${ }^{8} C_{i}^{*}=\operatorname{Enc}_{R_{E}}^{*}\left(0^{\left|\sigma_{*^{*}}^{\prime}\right|} ; \omega_{i}\right)$.

4. For $i \in[n]$, let $x_{i}$ denote the statement: " $\left(p k_{S, i}, M^{*}, R_{E}, C_{i}^{*}\right) \in L$ ", and let $x:=\bigvee_{i=1}^{n} x_{i}$. Compute the proof $\pi \leftarrow \mathcal{P}_{r_{1}}\left(x,\left(\sigma_{i^{*}}^{\prime}, \omega_{i^{*}}\right)\right)$.

5. The signature is $\sigma=\left(C_{1}^{*}, \ldots, C_{n}^{*}, \pi\right)$.

$\underline{\operatorname{Vrfy}_{P K_{1}, \ldots, P K_{n}}(M, \sigma)}$

1. Parse each $P K_{i}$ as $\left(p k_{S, i}, p k_{E, i}, r_{i}\right)$. Set $M^{*}:=M\left|P K_{1}\right| \cdots \mid P K_{n}$ and $R_{E}:=\left\{p k_{E, 1}, \ldots, p k_{E, n}\right\}$. Parse $\sigma$ as $\left(C_{1}^{*}, \ldots, C_{n}^{*}, \pi\right)$.

2. For $i \in[n]$, let $x_{i}$ denote the statement " $\left(p k_{S, i}, M^{*}, R_{E}, C_{i}^{*}\right) \in L$ " and set $x:=\bigvee_{i=1}^{n} x_{i}$.

3. Output $\mathcal{V}_{r_{1}}(x, \pi)$.

It is easy to see that the scheme above satisfies the functional definition of a ring signature scheme (recall that the $\left\{P K_{i}\right\}$ in a ring are always ordered lexicographically). We now prove that the scheme satisfies strong notions of anonymity and unforgeability:

Theorem 1. If encryption scheme (EGen, Enc, Dec) is semantically secure, signature scheme $\left(\mathrm{Gen}^{\prime}\right.$, Sign', $\left.\mathrm{Vrfy}^{\prime}\right)$ is existentially unforgeable under adaptive chosen-message attacks, and $(\ell, \mathcal{P}, \mathcal{V})$ is a $Z A P$ for the language $L^{\prime}=\left\{\left(x_{1}, \ldots\right.\right.$, $\left.\left.x_{n}\right): \exists i: x_{i} \in L\right\}$, then the above ring signature scheme is (computationally) anonymous against attribution attacks, and unforgeable w.r.t. insider corruption.

The proof is given in Appendix B.1.

Extension. The scheme above can also be used (with a few easy modifications) in a situation where some users in the ring have not generated a key pair according to Gen, as long as (1) every ring member has a public key both for encryption and for signing and (2) at least one of the members has included a sufficientlylong random string in his public key. Furthermore, the encryption (signature) public keys of different members of the ring may be associated with different encryption (signature) schemes. Thus, a single user who establishes a public key for a ring signature scheme suffices to provide anonymity for everyone. This also provides a way to include "oblivious" users in the signing ring $[1,2]$.

Achieving a stronger anonymity guarantee. The above scheme is not secure against full key exposure, and essential to our proof of anonymity is that the adversary not be given the random coins used to generate all (honest) ring

\footnotetext{
${ }^{8}$ We assume for simplicity that valid signatures w.r.t. the public keys $\left\{p k_{S, i}\right\}_{i \neq i^{*}}$ always have the same length as valid signatures w.r.t. $p k_{S, i^{*}}$. The construction can be adapted when this is not the case.
} 
signature keys. ${ }^{9}$ (If the adversary gets all sets of random coins, it can decrypt ciphertexts encrypted using Enc $_{R_{E}}^{*}$ for any ring of honest users $R$ and thereby determine the true signer of a message.) It is possible to achieve anonymity against full key exposure using an enhanced form of encryption for which, informally, there exists an "oblivious" way to generate a public key without generating a corresponding secret key. This notion, introduced by Damgård and Nielsen [10], can be viewed as a generalization of dense cryptosystems in which the public key is required to be a uniformly distributed string (in particular, dense cryptosystems satisfy the definition below). We review the formal definition here.

Definition 8. An oblivious key generator for the public-key encryption scheme (EGen, Enc, Dec) is a pair of PPT algorithms (ObIEGen, ObIRand) such that:

- ObIEGen, on input $1^{k}$ and random coins $\omega \in\{0,1\}^{n(k)}$, outputs a key $p k$;

- ObIRand, on input a key pk, outputs a string $\omega$;

and the following distribution ensembles are computationally indistinguishable:

$$
\left\{\omega \leftarrow\{0,1\}^{n(k)}:\left(\omega, \operatorname{ObIEGen}\left(1^{k} ; \omega\right)\right)\right\}
$$

and

$$
\left\{(p k, s k) \leftarrow \operatorname{EGen}\left(1^{k}\right) ; \omega \leftarrow \operatorname{ObIRand}(p k):(\omega, p k)\right\} .
$$

Note that if (EGen, Enc, Dec) is semantically secure, then (informally speaking) it is also semantically secure to encrypt messages using a public key $p k$ generated by ObIEGen, even if the adversary has the random coins used by ObIEGen in generating $p k$. We remark for completeness that the El Gamal encryption scheme (over the group of quadratic residues modulo a prime) is an example of a scheme having an oblivious key generator.

Given the above, we adapt our construction in the natural way: specifically, the Gen algorithm is changed so that instead of generating $p k_{E}$ using EGen (and then erasing the secret key $s k_{E}$ and the random coins used), we now generate $p k_{E}$ using OblEGen. Adapting the proof of Theorem 1, we can easily show:

Theorem 2. Under the assumptions of Theorem 1 and assuming (EGen, Enc, Dec) has an oblivious key generator, the modified ring signature scheme described above is (computationally) anonymous against full key exposure, and unforgeable w.r.t. insider corruption.

The proof is given in Appendix B.2.

\section{An Efficient 2-User Ring Signature Scheme}

In this section, we present a more efficient construction of a 2-user ring signature scheme based on specific assumptions. The scheme is based on the (standard) signature scheme constructed by Waters [21] which we briefly review now.

\footnotetext{
${ }^{9}$ We remark that anonymity still holds if the adversary is given all secret keys (but not the randomness used to generate all secret keys). This is because the decryption key $s k_{E}$ is erased, and not included in $S K$.
} 


\subsection{The Waters Scheme}

Let $\mathbb{G}, \mathbb{G}_{1}$ be groups of prime order $q$ such that there exists an efficiently computable bilinear map $\hat{e}: \mathbb{G} \times \mathbb{G} \rightarrow \mathbb{G}_{1}$. We assume that $q, \mathbb{G}, \mathbb{G}_{1}, \hat{e}$, and a generator $g \in \mathbb{G}$ are publicly known. The Waters signature scheme for messages of length $n$ is defined as follows:

Key Generation. Choose $\alpha \leftarrow \mathbb{Z}_{q}$ and set $g_{1}=g^{\alpha}$. Additionally choose random elements $h, u^{\prime}, u_{1}, \ldots, u_{n} \leftarrow \mathbb{G}$. The public key is $\left(g_{1}, h, u^{\prime}, u_{1}, \ldots, u_{n}\right)$ and the secret key is $h^{\alpha}$.

Signing. To sign the $n$-bit message $M$, first compute $w=u^{\prime} \cdot \prod_{i: M_{i}=1} u_{i}$. Then choose random $r \leftarrow \mathbb{Z}_{q}$ and output the signature $\sigma=\left(h^{\alpha} \cdot w^{r}, g^{r}\right)$.

Verification. To verify the signature $(A, B)$ on message $M$ with respect to public key $\left(g_{1}, h, u^{\prime}, u_{1}, \ldots, u_{n}\right)$, compute $w=u^{\prime} \cdot \prod_{i: M_{i}=1} u_{i}$ and then check whether $\hat{e}\left(g_{1}, h\right) \cdot \hat{e}(B, w) \stackrel{?}{=} \hat{e}(A, g)$.

\subsection{A 2-User Ring Signature Scheme}

The main observation we make with regard to the above scheme is the following: element $h$ is arbitrary, and only knowledge of $h^{\alpha}$ is needed to sign. So, we can dispense with including $h$ in the public key altogether; instead, a user $U$ with secret $\alpha$ and the value $g_{1}=g^{\alpha}$ in his public key will use as his " $h$-value" the value $\bar{g}_{1}$ contained in the public key of a second user $\bar{U}$. This provides anonymity since $\bar{U}$ could also have computed the same value $\left(\bar{g}_{1}\right)^{\alpha}$ using the secret value $\bar{\alpha}=\log _{g} \bar{g}_{1}$ known to him (because $\bar{g}_{1}^{\alpha}=g_{1}^{\bar{\alpha}}$ ). We now proceed with the details.

Key Generation. Choose $\alpha \leftarrow \mathbb{Z}_{q}$ and set $g_{1}=g^{\alpha}$. Additionally choose random elements $u^{\prime}, u_{1}, \ldots, u_{n} \leftarrow \mathbb{G}$. The public key is $\left(g_{1}, u^{\prime}, u_{1}, \ldots, u_{n}\right)$ and the secret key is $\alpha$. (We again assume that $q, \mathbb{G}, \mathbb{G}_{1}, \hat{e}$, and $g$ are system-wide parameters.)

Ring Signing. To sign message $M \in\{0,1\}^{n}$ with respect to the ring $R=$ $\{P K, \overline{P K}\}$ using secret key $\alpha$ (where we assume without loss of generality that $\alpha$ is the secret corresponding to $P K)$, proceed as follows: parse $P K$ as $\left(g_{1}, u^{\prime}\right.$, $\left.u_{1}, \ldots, u_{n}\right)$ and $\overline{P K}$ as $\left(\bar{g}_{1}, \bar{u}^{\prime}, \bar{u}_{1}, \ldots, \bar{u}_{n}\right)$, and compute $w=u^{\prime} \cdot \prod_{i: M_{i}=1} u_{i}$ and $\bar{w}=\bar{u}^{\prime} \cdot \prod_{i: M_{i}=1} \bar{u}_{i}$. Then choose random $r \leftarrow \mathbb{Z}_{q}$ and output the signature

$$
\sigma=\left(\bar{g}_{1}^{\alpha} \cdot(w \bar{w})^{r}, g^{r}\right)
$$

Ring Verification. To verify the signature $(A, B)$ on message $M$ with respect to the ring $R=\{P K, \overline{P K}\}$ (parsed as above), compute $w=u^{\prime} \cdot \prod_{i: M_{i}=1} u_{i}$ and $\bar{w}=\bar{u}^{\prime} \cdot \prod_{i: M_{i}=1} \bar{u}_{i}$ and then check whether $\hat{e}\left(g_{1}, \bar{g}_{1}\right) \cdot \hat{e}(B,(w \bar{w})) \stackrel{?}{=} \hat{e}(A, g)$.

It is not hard to see that correctness holds. We prove the following regarding the above scheme: 
Theorem 3. Assume the Waters signature scheme is existentially unforgeable ${ }^{10}$ under adaptive chosen message attack. Then the 2-user ring signature scheme described above is unconditionally anonymous against full key exposure, and unforgeable against chosen-subring attacks.

Proof. Unconditional anonymity against full key exposure follows easily from the observation made earlier: namely, that only the value $\bar{g}_{1}^{\alpha}=g_{1}^{\bar{\alpha}}$ (where $\bar{\alpha} \stackrel{\text { def }}{=}$ $\log _{g} \bar{g}_{1}$ ) is needed to sign, and either of the two (honest) parties can compute this value.

We now prove that the scheme satisfies Definition 6 . We do this by showing how an adversary $\mathcal{A}$ that forges a signature with respect to the ring signature scheme with non-negligible probability can be used to construct an adversary $\hat{\mathcal{A}}$ that forges a signature with respect to the Waters signature scheme (in the standard sense) with the same probability. For simplicity in the proof, we assume that $\mathcal{A}$ only ever sees the public keys of two users, requests all signatures to be signed with respect to the ring $R$ containing these two users, and forges a signature with respect to that same ring $R$. By a hybrid argument, it can be shown that (for this scheme) this is equivalent to the more general case when $\mathcal{A}$ may see multiple public keys, request signatures with respect to various (different) 2-user subsets, and then output a forgery with respect to any 2-user subset of its choice.

Construct $\hat{\mathcal{A}}$ as follows: $\hat{\mathcal{A}}$ is given the public key $\left(\hat{g}_{1}, \hat{h}, \hat{u}^{\prime}, \hat{u}_{1}, \ldots, \hat{u}_{n}\right)$ of an instance of the Waters scheme. $\hat{\mathcal{A}}$ constructs two user public keys as follows: first, it sets $g_{1}=\hat{g}_{1}$ and $\bar{g}_{1}=\hat{h}$. Then, it chooses random $u^{\prime}, u_{1}, \ldots, u_{n} \leftarrow \mathbb{G}$ and sets $\bar{u}^{\prime}=\hat{u}^{\prime} / u^{\prime}$ and $\bar{u}_{i}=\hat{u}_{i} / u_{i}$ for all $i$. It gives to $\mathcal{A}$ the public keys $\left(g_{1}, u^{\prime}, u_{1}\right.$, $\left.\ldots, u_{n}\right)$ and $\left(\bar{g}_{1}, \bar{u}^{\prime}, \bar{u}_{1}, \ldots, \bar{u}_{n}\right)$. Note that both public keys have the appropriate distribution. When $\mathcal{A}$ requests a ring signature on a message $M$ with respect to the ring $R$ containing these two public keys, $\hat{\mathcal{A}}$ requests a signature on $M$ from its signing oracle, obtains in return a signature $(A, B)$, and gives this signature to $\mathcal{A}$. Note that this is indeed a perfect simulation, since

$$
\left(\hat{h}^{\log _{g} \hat{g}_{1}} \cdot\left(\hat{u}^{\prime} \prod_{i: M_{i}=1} \hat{u}_{i}\right)^{r}, g^{r}\right)=\left(\bar{g}_{1}^{\log _{g} g_{1}} \cdot\left(u^{\prime} \bar{u}^{\prime} \prod_{i: M_{i}=1} u_{i} \bar{u}_{i}\right)^{r}, g^{r}\right),
$$

which is an appropriately-distributed ring signature with respect to the public keys given to $\mathcal{A}$.

When $\mathcal{A}$ outputs a forgery $\left(A^{*}, B^{*}\right)$ on a message $M^{*}$, this same forgery is output by $\hat{\mathcal{A}}$. Note that $\hat{\mathcal{A}}$ outputs a valid forgery whenever $\mathcal{A}$ does, since

$$
\hat{e}\left(g_{1}, \bar{g}_{1}\right) \cdot \hat{e}\left(B^{*},\left(u^{\prime} \bar{u}^{\prime} \prod_{i: M_{i}^{*}=1} u_{i} \bar{u}_{i}\right)\right)=\hat{e}\left(A^{*}, g\right)
$$

implies

$$
\hat{e}\left(\hat{g}_{1}, \hat{h}\right) \cdot \hat{e}\left(B^{*},\left(\hat{u}^{\prime} \prod_{i: M_{i}^{*}=1} \hat{u}_{i}\right)\right)=\hat{e}\left(A^{*}, g\right) .
$$

$\overline{10 \text { This holds }}[21]$ under the computational Diffie-Hellman assumption in $\mathbb{G}$. 
We conclude that $\hat{\mathcal{A}}$ outputs a forgery with the same probability as $\mathcal{A}$. Since, by assumption, the Waters scheme is secure, this completes the proof.

We remark that the security reduction in the above proof is tight.

An efficiency improvement. A (slightly) more efficient variant of the above scheme is also possible. Key generation is the same as before, except that an additional, random identifier $I \in\{0,1\}^{k}$ is also chosen and included in the public key. Let $<_{\text {lex }}$ denote lexicographic order. To sign message $M \in\{0,1\}^{n}$ with respect to the ring $R=\{P K, \overline{P K}\}$, first parse $P K$ as $\left(I, g_{1}, u^{\prime}, u_{1}, \ldots, u_{n}\right)$ and $\overline{P K}$ as $\left(\bar{I}, \bar{g}_{1}, \bar{u}^{\prime}, \bar{u}_{1}, \ldots, \bar{u}_{n}\right)$. Choose random $r \leftarrow \mathbb{Z}_{q}$. If $I \leq_{\operatorname{lex}} \bar{I}$, compute $w=u^{\prime} \cdot \prod_{i: M_{i}=1} u_{i}$ and the signature

$$
\sigma=\left(s \cdot w^{r}, g^{r}\right)
$$

if $\bar{I}<_{\text {lex }} I$, compute $\bar{w}=\bar{u}^{\prime} \cdot \prod_{i: M_{i}=1} \bar{u}_{i}$ and the signature

$$
\sigma=\left(s \cdot \bar{w}^{r}, g^{r}\right)
$$

where, in each case, $s=\bar{g}_{1}^{\alpha}=g_{1}^{\bar{\alpha}}$ is computed using whichever secret key is known to the signer. Verification is changed in the obvious way. A proof similar to the above shows that this scheme satisfies the same security properties as in Theorem 3 .

\section{References}

1. M. Abe, M. Ohkubo, and K. Suzuki. 1-out-of-n signatures from a variety of keys. In Advances in Cryptology - Asiacrypt 2002.

2. B. Adida, S. Hohenberger, and R.L. Rivest. Ad-hoc-group signatures from hijacked

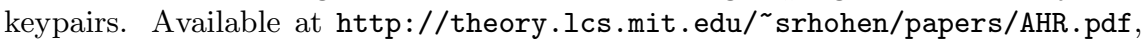
2005.

3. M. Bellare, D. Micciancio, and B. Warinschi. Foundations of group signatures: Formal definitions, simplified requirements, and a construction based on general assumptions. In Advances in Cryptology - Eurocrypt 2003.

4. A. Bender, J. Katz, and R. Morselli. Ring signatures: Stronger definitions, and constructions without random oracles. Cryptology ePrint Archive, 2005. http://eprint.iacr.org/2005/304.

5. E. Bresson, J. Stern, and M. Szydlo. Threshold ring signatures and applications to ad-hoc groups. In Advances in Cryptology - Crypto 2002.

6. D. Chaum and E. van Heyst. Group signatures. In Advances in Cryptology Eurocrypt '91.

7. L. Chen, C. Kudla, and K.G. Patterson. Concurrent signatures. In Advances in Cryptology - Eurocrypt 2004.

8. S. S.M. Chow, J.K. Liu, and T. H. Yuen. Ring signature without random oracles. Cryptology ePrint Archive, 2005. http://eprint.iacr.org/2005/317.

9. R. Cramer, I. Damgård, and B. Schoenmakers. Proofs of partial knowledge and simplified design of witness hiding protocols. In Advances in Cryptology - Crypto $' 94$. 
10. I. Damgård and J.B. Nielsen. Improved non-committing encryption schemes based on a general complexity assumption. In Advances in Cryptology - Crypto 2000.

11. Y. Dodis, A. Kiayias, A. Nicolosi, and V. Shoup. Anonymous identification in ad-hoc groups. In Advances in Cryptology - Eurocrypt 2002.

12. C. Dwork and M. Naor. Zaps and their applications. In Proc. 41st Annual Symposium on Foundations of Computer Science (FOCS). IEEE, 2000.

13. U. Feige, D. Lapidot, and A. Shamir. Multiple non-interactive zero knowledge proofs under general assumptions. SIAM J. Computing, 29(1):1-28, 1999.

14. A. Fiat and A. Shamir. How to prove yourself: Practical solutions to identification and signature problems. In Advances in Cryptology - Crypto ' 86.

15. J. Herranz. Some digital signature schemes with collective signers. $\mathrm{PhD}$ thesis, Universitat Politècnica de Catalunya, Barcelona, April 2005. Available at http://www.lix.polytechnique.fr/ ${ }^{\sim}$ herranz/thesis.htm.

16. J. Herranz and G. Sáez. Forking lemmas for ring signature schemes. In Progress in Cryptology - Indocrypt 2003.

17. M. Jakobsson, K. Sako, and R. Impagliazzo. Designated verifier proofs and their applications. In Advances in Cryptology - Eurocrypt '96.

18. J.K. Liu, V.K. Wei, and D.S. Wong. Linkable spontaneous anonymous group signatures for ad hoc groups. In ACISP 2004.

19. M. Naor. Deniable ring authentication. In Advances in Cryptology - Crypto 2002.

20. R.L. Rivest, A. Shamir, and Y. Tauman. How to leak a secret. In Asiacrypt 2001. Full version available at http://www.mit.edu/ ${ }^{\sim}$ tauman and to appear in Essays in Theoretical Computer Science: in Memory of Shimon Even.

21. B. Waters. Efficient identity-based encryption without random oracles. In Advances in Cryptology - Eurocrypt 2005.

22. J. Xu, Z. Zhang, and D. Feng. A ring signature scheme using bilinear pairings. In Workshop on Information Security Applications (WISA), 2004.

23. F. Zhang and K. Kim. ID-based blind signature and ring signature from pairings. In Advances in Cryptology - Asiacrypt 2002.

\section{A ZAPs}

Let $L$ be an $\mathcal{N P}$ language with associated polynomial-time and polynomiallybounded witness relation $\mathcal{R}_{L}$ (i.e., such that $L \stackrel{\text { def }}{=}\left\{x \mid \exists w:(x, w) \in \mathcal{R}_{L}\right\}$ ). If $(x, w) \in \mathcal{R}_{L}$ we refer to $x$ as the statement and $w$ as the associated witness for $x$. We now recall the definition of a ZAP from [12]:

Definition 9 (ZAP). A ZAP for an $\mathcal{N P}$ language $L$ (with associated witness relation $\left.\mathcal{R}_{L}\right)$ is a triple $(\ell, \mathcal{P}, \mathcal{V})$, where $\ell(\cdot)$ is a polynomial, $\mathcal{P}$ is a PPT algorithm, and $\mathcal{V}$ is polynomial-time deterministic algorithm, and such that.

Completeness For $^{11}$ any $(x, w) \in \mathcal{R}_{L}$ and any $r \in\{0,1\}^{\ell(k)}$ :

$$
\operatorname{Pr}\left[\pi \leftarrow \mathcal{P}_{r}(x, w): \mathcal{V}_{r}(x, \pi)=1\right]=1 .
$$

${ }^{11}$ We remark that the definition in [12] allows for a negligible completeness error. However, their construction achieves perfect completeness when instantiated using the NIZK of [13]. 
Adaptive soundness There exists a negligible function $\varepsilon$ such that

$$
\operatorname{Pr}\left[r \leftarrow\{0,1\}^{\ell(k)}: \exists(x, \pi): \quad x \notin L \text { and } \mathcal{V}_{r}(x, \pi)=1\right] \leq \varepsilon(k) .
$$

Witness indistinguishability (Informal) For any $x \in L$, any pair of witnesses $w_{0}, w_{1}$ for $x$, and any $r \in\{0,1\}^{\ell(k)}$, the distributions $\left\{\mathcal{P}_{r}\left(x, w_{0}\right)\right\}$ and $\left\{\mathcal{P}_{r}\left(x, w_{1}\right)\right\}$ are computationally indistinguishable. (Note: more formally, we need to speak in terms of sequences $\left\{r_{k} \in\{0,1\}^{\ell(k)}\right\},\left\{x_{k}\right\}$, and $\left\{\left(w_{k, 0}, w_{k, 1}\right)\right\}$ but we avoid doing so for simplicity of exposition.)

A ZAP is used in the following way: The verifier generates a random first message $r \leftarrow\{0,1\}^{\ell(k)}$ and sends it to the prover $\mathcal{P}$. The prover, given $r$, a statement $x$, and associated witness $w$, sends $\pi \leftarrow \mathcal{P}_{r}(x, w)$ to the verifier. The verifier then runs $\mathcal{V}_{r}(x, \pi)$ and accepts iff the output is 1.

\section{B Proofs of Theorems 1 and 2}

\section{B.1 Proof of Theorem 1}

We restate Theorem 1 for convenience:

If encryption scheme (EGen, Enc, Dec) is semantically secure, signature scheme $\left(\mathrm{Gen}^{\prime}\right.$, Sign', Vrfy') is existentially unforgeable under adaptive chosen-message attacks, and $(\ell, \mathcal{P}, \mathcal{V})$ is a ZAP for $L$ as described above, then the above ring signature scheme is (computationally) anonymous against attribution attacks, and unforgeable w.r.t. insider corruption.

Proof. We prove each of the desired security properties in turn.

Anonymity. For simplicity of exposition, we consider Definition 4 with $n=2$; i.e., we assume only two users. By a straightforward hybrid argument, this implies the general case. Given a PPT adversary $\mathcal{A}$, we consider a sequence of experiments $E_{0}$, Hybrid $_{0}$, Hybrid $_{1}, E_{1}$ such that $E_{0}$ (resp., $E_{1}$ ) corresponds to the experiment of Definition 4 with $b=0$ (resp., $b=1$ ), and such that each experiment is computationally indistinguishable from the one before it. This implies that $\mathcal{A}$ has negligible advantage in distinguishing $E_{0}$ from $E_{1}$, as desired.

For convenience, we review experiment $E_{0}$. Here, two key pairs $\left(P K_{0}=\right.$ $\left.\left(p k_{S, 0}, p k_{E, 0}, r_{0}\right), S K_{0}\right)$ and $\left(P K_{1}=\left(p k_{S, 1}, p k_{E, 1}, r_{1}\right), S K_{1}\right)$ are generated and $\mathcal{A}$ is given $P K_{0}$ and the randomness used to generate $\left(P K_{1}, S K_{1}\right)$ (by hybrid argument, we can assume that $i_{0}=0$ and $\left.i_{1}=1\right)$. The adversary is also given access to a signing oracle (which can be used to obtain signatures computed using $\left.S K_{0}\right)$. $\mathcal{A}$ then outputs a message $M$ along with a ring of public keys $R$ containing both $P K_{0}$ and $P K_{1}$. Finally, $\mathcal{A}$ is given $\sigma \leftarrow \operatorname{Sign}_{S K_{0}}(M, R)$.

Experiment Hybrid $_{0}$ is the same as experiment $E_{0}$ except that we change how the signature $\sigma$ is generated. In particular, step 3 of the ring signing algorithm is modified as follows: let $R_{E}$ and $M^{*}$ be as in the description of the ring signing algorithm given earlier. In step 3 , instead of setting $C_{1}^{*}$ to be an encryption of all 
zeros, we now compute $\sigma_{1}^{\prime} \leftarrow \operatorname{Sign}_{s k_{S, 1}}\left(M^{*}\right)$ and then set $C_{1}^{*}=\operatorname{Enc}_{R_{E}}^{*}\left(\sigma_{1}^{\prime} ; \omega_{1}\right)$. We stress that, as in $E_{0}$, the ciphertext $C_{0}^{*}$ is still set to be an encryption of the signature $\sigma_{0}^{\prime}$, and the remaining ciphertexts are still encryptions of all zeros.

It is not hard to see that experiment Hybrid $_{0}$ is computationally indistinguishable from experiment $E_{0}$, assuming semantic security of the encryption scheme (EGen, Enc, Dec). This follows from the observations that (1) adversary $\mathcal{A}$ is not given the random coins used in generating $P K_{0}$ and so, in particular, it is not given the coins used to generate $p k_{E, 0} ;(2)$ (informally) semantic security of encryption under $\mathrm{Enc}_{p k_{E, 0}}$ implies semantic security of encryption using $\mathrm{Enc}_{R_{E}}^{*}$ as long as $p k_{E, 0} \in R_{E}$ (a formal proof is straightforward); and, finally, (3) the coins $\omega_{1}$ used in generating $C_{1}^{*}$ are not used in the remainder of the ring signing algorithm.

Experiment Hybrid $_{1}$ is the same as Hybrid ${ }_{0}$ except that we use a different witness when computing the proof $\pi$ for the ZAP. In particular, instead of using witness $\left(\sigma_{0}^{\prime}, \omega_{0}\right)$ we use the witness $\left(\sigma_{1}^{\prime}, \omega_{1}\right)$. The remainder of the signing algorithm is unchanged.

It is relatively immediate that experiment Hybrid $_{1}$ is computationally indistinguishable from Hybrid $_{0}$, assuming witness indistinguishability of the ZAP. (We remark that the use of a ZAP, rather than non-interactive zero-knowledge, is essential here since the adversary may choose the "random string" component of all the adversarially-chosen public keys any way it likes.) In more detail, we can construct the following malicious verifier algorithm $\mathcal{V}^{*}$ using $\mathcal{A}$ : verifier $\mathcal{V}^{*}$ generates $\left(P K_{0}, S K_{0}\right)$ and $\left(P K_{1}, S K_{1}\right)$ exactly as in experiments Hybrid and $_{0}$ Hybrid $_{1}$, and gives these keys and the appropriate associated random coins to $\mathcal{A}$. The signing queries of $\mathcal{A}$ can easily be answered by $\mathcal{V}^{*}$. When $\mathcal{A}$ makes its signing query, $\mathcal{V}^{*}$ computes the $C_{i}^{*}$ exactly as in Hybrid $_{1}$ and then gives to the prover $\mathcal{P}$ the keys $\left\{p k_{S, i}\right\}_{i \in R}$, the message $M^{*}$, the set of keys $R_{E}$, and the ciphertexts $\left\{C_{i}^{*}\right\}_{i \in R}$; this defines the $\mathcal{N} \mathcal{P}$-statement $x$ exactly as in step 4 of the ring signing algorithm. In addition, $\mathcal{V}^{*}$ gives the two witnesses $\left(\sigma_{0}^{\prime}, \omega_{0}\right)$ and $\left(\sigma_{1}^{\prime}, \omega_{1}\right)$ to $\mathcal{P}$. Finally, $\mathcal{V}^{*}$ sends as its first message the "random string" component $r$ of the lexicographically-first public key in $R$ (note that this $r$ is the random string that would be used to generate the proof $\pi$ in step 4 of the ring signing algorithm). The prover responds with a proof $\pi \leftarrow \mathcal{P}_{r}\left(x,\left(\sigma_{b}^{\prime}, \omega_{b}\right)\right)$ (for some $b \in\{0,1\}$ ), and then $\mathcal{V}^{*}$ outputs $\left(C_{1}^{*}, \ldots, C_{n}^{*}, \pi\right)$.

Note that if the prover uses the first witness provided to it by $\mathcal{V}^{*}$ then the output of $\mathcal{V}^{*}$ is distributed exactly according to Hybrid 0 , while if the prover uses the second witness provided to it by $\mathcal{V}^{*}$ then the output of $\mathcal{V}^{*}$ is distributed exactly according to Hybrid $_{1}$. Witness indistinguishability of the ZAP thus implies computational indistinguishability of $\mathrm{Hybrid}_{0}$ and Hybrid ${ }_{1}$.

We may now notice that Hybrid $_{1}$ is computationally indistinguishable from $E_{1}$ by exactly the same argument used to show the indistinguishability of $\mathrm{Hybrid}_{0}$ and $E_{0}$. This completes the proof.

Unforgeability. Assume there exists a PPT adversary $\mathcal{A}$ that breaks the above ring signature scheme (in the sense of Definition 7) with non-negligible probability. We construct an adversary $\mathcal{A}^{\prime}$ that breaks the underlying signature scheme 
(Gen', Sign', Vrfy') (in the standard sense of existential unforgeability) with nonnegligible probability.

$\mathcal{A}^{\prime}$ receives as input a public key $p k_{S}$. Let $n=n(k)$ be a bound on the number of (honest user) public keys that $\mathcal{A}$ expects to be generated. $\mathcal{A}^{\prime}$ runs $\mathcal{A}$ with input public keys $S=\left\{P K_{1}, \ldots, P K_{n}\right\}$, that $\mathcal{A}^{\prime}$ generates as follows. $\mathcal{A}^{\prime}$ chooses $i^{*} \leftarrow\{1, \ldots, n\}$ and sets $p k_{S, i^{*}}=p k_{S}$. The remainder of public key $P K_{i^{*}}$ is generated exactly as prescribed by the Gen algorithm, with the exception that the decryption key $s k_{E, i^{*}}$ that is generated is not erased. Public keys $P K_{i}$ for $i \neq i^{*}$ are also generated exactly as prescribed by the Gen algorithm, again with the exception that the decryption keys $\left\{s k_{E, i}\right\}$ are not erased.

$\mathcal{A}^{\prime}$ then proceeds to simulate the oracle queries of $\mathcal{A}$ in the natural way:

1. When $\mathcal{A}$ requests a signature on message $M$, with respect to ring $R$ (which may possibly contain some public keys generated in an arbitrary manner by $\mathcal{A}$ ), to be signed by user $i \neq i^{*}$, then $\mathcal{A}^{\prime}$ can easily generate the response to this query by running the Sign algorithm completely honestly;

2. When $\mathcal{A}$ requests a signature on message $M$, with respect to $\operatorname{ring} R$ (which, again, may possibly contain some public keys generated in an arbitrary manner by $\mathcal{A}$ ) to be signed by user $i^{*}$, then $\mathcal{A}^{\prime}$ cannot directly respond to this query since it does not have $s k_{S, i^{*}}$. Instead, $\mathcal{A}^{\prime}$ sets $M^{*}$ appropriately, submits $M^{*}$ to its signing oracle, and obtains in return a signature $\sigma_{i^{*}}^{\prime}$. It then computes the remainder of the ring signature by following the rest of the Sign algorithm; note, in particular, that $s k_{S, i^{*}}$ is not needed for this;

3 . Any corruption query made by $\mathcal{A}$ for a user $i \neq i^{*}$ can be faithfully answered by $\mathcal{A}^{\prime}$. On the other hand, if $\mathcal{A}$ ever makes a corruption query for $i^{*}$, then $\mathcal{A}^{\prime}$ simply aborts.

At some point, $\mathcal{A}$ outputs a forgery $\bar{\sigma}=\left(\bar{C}_{1}^{*}, \ldots, \bar{C}_{n}^{*}, \bar{\pi}\right)$ on a message $\bar{M}$ with respect to some ring of honest-user public keys $\bar{R} \subseteq S$. If $P K_{i^{*}} \notin \bar{R}$, then $\mathcal{A}^{\prime}$ aborts. Otherwise, since $\mathcal{A}^{\prime}$ knows all relevant decryption keys (recall that the ring $\bar{R}$ contains public keys of honest users only, and these keys were generated by $\left.\mathcal{A}^{\prime}\right)$ it can decrypt $\bar{C}_{i^{*}}^{*}$ and obtain a candidate signature $\bar{\sigma}_{i^{*}}$. Finally, $\mathcal{A}^{\prime}$ sets $\bar{M}^{*}=\bar{M}\left|\overline{P K}_{1}\right| \cdots \mid \overline{P K}_{n^{\prime}}$ (where $\bar{R}=\left\{\overline{P K}_{i}\right\}$ ) and outputs $\left(\bar{M}^{*}, \bar{\sigma}_{i^{*}}\right)$. Note that (by requirement) $\mathcal{A}$ never requested a signature on message $\bar{M}$ with respect to the ring $\bar{R}$, and so $\mathcal{A}^{\prime}$ never requested a signature on message $\bar{M}^{*}$ from its own oracle.

We claim that if $\mathcal{A}$ forges a signature with non-negligible probability $\varepsilon=\varepsilon(k)$, then $\mathcal{A}^{\prime}$ forges a signature with probability at least $\varepsilon^{\prime}=\varepsilon / n-\operatorname{negl}(k)$. To see this, note first that if $\mathcal{A}$ outputs a valid forgery then with all but negligible probability (by soundness of the ZAP) it holds that $\left(\overline{p k}_{S, i}, \bar{M}^{*}, \bar{R}_{E}, \bar{C}_{i}^{*}\right) \in L$ for some $i$ (where $\overline{p k}_{S, i}$ and $\bar{R}_{E}$ are defined in the natural way based on the ring $\bar{R}$ and the public keys it contains). Conditioned on this, with probability $1 / n$ it is the case that (1) $\mathcal{A}^{\prime}$ did not abort and furthermore (2) $\left(\overline{p k}_{S, i^{*}}, \bar{M}^{*}, \bar{R}_{E}, \bar{C}_{i^{*}}^{*}\right) \in L$. When this occurs, then with all but negligible probability $\mathcal{A}^{\prime}$ will recover (by decrypting as described above) a valid signature $\bar{\sigma}_{i^{*}}$ on the message $\bar{M}^{*}$ with respect to the given public key $\overline{p k}_{S, i^{*}}=p k_{S}$ (relying here on the fact that with all but negligible 
probability over choice of encryption public keys the encryption scheme Enc* has zero decryption error). Security of $\left(\mathrm{Gen}^{\prime}, \mathrm{Sign}^{\prime}, \mathrm{Vrfy}{ }^{\prime}\right)$ thus implies that $\varepsilon$ must be negligible.

\section{B.2 Proof of Theorem 2}

We restate Theorem 2 for convenience:

Under the assumptions of Theorem 1 and assuming (EGen, Enc, Dec) has an oblivious key generator, the modified ring signature scheme described above is (computationally) anonymous against full key exposure, and unforgeable w.r.t. insider corruption.

Proof. The proof of unforgeability follows immediately from Theorem 1 since, by Definition 8 , the adversary cannot distinguish between the original scheme (in which the encryption key is generated using EGen) and the modified scheme (in which the encryption key is generated using ObIEGen).

We now argue that the modified scheme achieves anonymity against full key exposure. First we note that the anonymity against attribution attacks claimed in Theorem 1 holds even when the adversary is given all random coins used to generate $\left(P K_{0}, S K_{0}\right)$ except for those coins used to generate $p k_{E, 0}$ (using EGen). Now, if there exists a PPT adversary $\mathcal{A}$ that breaks anonymity of the modified scheme in the sense of full key exposure, we can use it to construct a PPT adversary $\mathcal{A}^{\prime}$ that breaks anonymity of the original scheme against attribution attacks. $\mathcal{A}^{\prime}$ receives $P K_{0}$, the random coins $\omega_{S, 1}, \omega_{E, 1}$ used to generate $\left(P K_{1}, S K_{1}\right)$, and the random coins $\omega_{S, 0}$ used to generate $p k_{S, 0}$ (i.e., $\mathcal{A}$ is not given the coins used to generate $\left.p k_{E, 0}\right)$. Next, $\mathcal{A}^{\prime}$ runs $\omega_{E, 0}^{\prime} \leftarrow \operatorname{ObIRand}\left(p k_{E, 0}\right)$ and $\omega_{E, 1}^{\prime} \leftarrow \operatorname{ObIRand}\left(p k_{E, 1}\right)$ and gives to $\mathcal{A}$ the public key $P K_{0}$ it received as well as the random coins $\omega_{S, 0}, \omega_{E, 0}^{\prime}, \omega_{S, 1}, \omega_{E, 1}^{\prime}$. The remainder of $\mathcal{A}$ 's execution is simulated in the natural way by $\mathcal{A}^{\prime}$.

Now, Definition 8 implies that the advantage of $\mathcal{A}$ in the above is negligibly close to the advantage of $\mathcal{A}$ in attacking the modified scheme in the sense of full key exposure. But the advantage of $\mathcal{A}$ in the above is exactly the advantage of $\mathcal{A}^{\prime}$ in attacking the original scheme via key attribution attack. Since we have already proved that the original scheme is anonymous against attribution attacks (cf. Theorem 1), we see that the modified scheme is anonymous against full key exposure. 